\title{
Aging of the Zero-Field-Cooled Magnetization in Ising Spin Glasses: Experiment and Numerical Simulation
}

\author{
Lorenzo W. Bernardi ${ }^{1}$, Hajime Yoshino ${ }^{1}$, Koji Hukushima ${ }^{1}$, Hajime Takayama ${ }^{1}$, Aya Tobo $^{2}$, and Atsuko Ito ${ }^{3}$ \\ 1 Institute for Solid State Physics, University of Tokyo, Kashiwa, 277-8581, Japan \\ ${ }^{2}$ Institute of Materials Research, Tohoku University, Sendai 980-8577, Japan \\ ${ }^{3}$ The institute of Physical and Chemical Research (RIKEN), Wako, Saitama, 351-0198, Japan
}

(May 30, 2000)

\begin{abstract}
A new protocol of the zero-field-cooled (ZFC) magnetization process is studied experimentally on an Ising spin-glass (SG) $\mathrm{Fe}_{0.50} \mathrm{Mn}_{0.50} \mathrm{TiO}_{3}$ and numerically on the Edwards-Anderson Ising SG model. Although the time scales differ very much between the experiment and the simulation, the behavior of the ZFC magnetization observed in the two systems can be interpreted by means of a common scaling expression based on the droplet picture. The results strongly suggest that the SG coherence length, or the mean size of droplet excitations, involved even in the experimental ZFC process, is about a hundred lattice distances or less.
\end{abstract}

PACS numbers: 75.10.Nr, 75.40.Gb, 75.50.Lk

Spin glasses generally exhibit puzzling aging behavior such that their magnetic response in the spin-glass (SG) phase below its transition temperature $T_{\mathrm{c}}$ depends crucially on their thermal history within the phase. For example, an isothermal zero-field-cooled (ZFC) magnetization, $m_{\mathrm{ZFC}, T}\left(t_{a}, t_{\mathrm{w}}\right)$, is defined as the magnetization induced by a magnetic field $h$ which is applied after a system is rapidly quenched from above $T_{\mathrm{c}}$ to a temperature $T$ below $T_{\mathrm{c}}$ and is aged for a waiting time of $t_{\mathrm{w}}$, and $t_{a}=\tau+t_{\mathrm{w}}$ with $\tau$ being the time that the system ages at $T$ under $h$. With increasing $\tau, m_{\mathrm{ZFC}, T}\left(t_{a}, t_{\mathrm{w}}\right)$ grows slowly. In contrast to ideal equilibrium situations, however, it depends not only on $\tau$ but also on the waiting time $t_{\mathrm{w}}$, namely, the larger $t_{\mathrm{w}}$, the slower the growth of $m_{\mathrm{ZFC}, T}\left(t_{a}, t_{\mathrm{w}}\right)$. This was the first observation of aging phenomena in spin glasses [1]. Since then most of the studies have focused on its $t_{\mathrm{w}}$-dependence.

In the present work, on the other hand, we have studied the ZFC magnetization focusing on its dependence on thermal processes after the field $h$ is applied. We denote it by $m_{\mathrm{ZFC}, T[\tau]}\left(t_{a}, t_{\mathrm{w}}\right)$ where $t_{\mathrm{w}}$ symbolically represents a common thermal history before $h$ is applied, while $T[\tau]$ does various heating processes after $h$ is switched on, e.g., with a fixed heating rate but with an intermittent stop(s) at a different temperature(s). We have carried out this new protocol of the ZFC magnetization measurement both experimentally on an Ising SG material $\mathrm{Fe}_{0.50} \mathrm{Mn}_{0.50} \mathrm{TiO}_{3}$ [2], hereafter referred to as FeMnTiO, and numerically on the 3D Gaussian Edwards-Anderson (EA) Ising SG model. Quite interestingly, we have found that $m_{\mathrm{ZFC}, T[\tau]}\left(t_{a}, t_{\mathrm{w}}\right)$ measured in the two systems are well interpreted by a unified way, or more explicitly, they are described by a common scaling expression which can be derived based on the droplet picture for the SG phase [3]. The purpose of the present letter is to demonstrate this finding and to discuss its implications on the aging dynamics in spin glasses.

According to the droplet picture, the mean size, $R\left(t_{\mathrm{w}}\right)$, of domains of an equilibrium state grows continuously when a spin glass relaxes with increasing $t_{\mathrm{w}}$. In fact its growth law in isothermal aging at a temperature $T$, denoted by $R_{T}\left(t_{\mathrm{w}}\right)$, has been numerically studied by the various groups [4], and within the numerical accuracy all the results are consistent with each other. Here we quote our result [7],

$$
R_{T}\left(t_{\mathrm{w}}\right) \simeq\left(t_{\mathrm{w}} / \tau_{0}\right)^{b_{\mathrm{f}} T / T_{\mathrm{c}}}
$$

where $\tau_{0}$ is the microscopic unit of time which is one Monte Carlo step (MCS) in the simulation, and $b_{\mathrm{f}} \simeq$ $0.17 T_{\mathrm{c}} / J$ [7] $\simeq 0.153$ with $T_{\mathrm{c}} \simeq 0.9 \mathrm{~J}[8], J$ being the variance of the interactions. Furthermore we have shown [9] that the aging properties of the spin auto-correlation function are consistently explained when the same functional form is adopted for the maximum size of droplets which can be activated within a time scale of $\tau, L_{T}(\tau)$, i.e.,

$$
L_{T}(\tau) \simeq\left(\tau / \tau_{0}\right)^{b_{\mathrm{f}} T / T_{\mathrm{c}}}
$$

with the same constant $b_{\mathrm{f}}$. For an aging process in which the temperature is changed as in our new protocol, the maximum size of droplet excitations at time $t_{a}=\tau+t_{\mathrm{w}}$, denoted by $L(\tau)$, is evaluated by the successive use of eq.(2) as described by eqs.(田) and (5) below.

In terms of $L(\tau)$ introduced just above the common scaling expression we have found is written as

$$
h^{-1} m_{\mathrm{ZFC}, T[\tau]}\left(t_{a}, t_{\mathrm{w}}\right)=\chi_{\mathrm{ZFC}}\left(L(\tau), R\left(t_{\mathrm{w}}\right)\right),
$$

where $R\left(t_{\mathrm{w}}\right)$ is the mean domain size at the instance when the field $h$ is switched on. For isothermal processes under a field within the linear response regime the droplet theory [3] has already predicted a similar scaling form, i.e., $m_{\mathrm{ZFC}, T}\left(t_{a}, t_{\mathrm{w}}\right) / \mathrm{h}$ is a function of $R_{T}\left(t_{\mathrm{w}}\right)$ and $L_{T}(\tau)$. Equation (3) extends it to aging processes which involve temperature changes. Also, by the low temperature expansion of the droplet theory, $m_{\mathrm{ZFC}, T}\left(t_{a}, t_{\mathrm{w}}\right) / \mathrm{h}$ in the quasi-equilibrium regime with $R_{T}\left(t_{\mathrm{w}}\right) \gg L_{T}(\tau)$ is shown [3.9] not to depend explicitly on $T$. Equation (3) 
shares the same property but for the whole range of $\tau$. In relation to this it is worth pointing out that the fieldcooled (FC) magnetization, $m_{\mathrm{FC}}(T)$, which is supposed to be $\lim _{t_{a} \rightarrow \infty} m_{\mathrm{ZFC}, T}\left(t_{a}, t_{\mathrm{w}}\right)$, is nearly independent of $T$ for the Ising spin glasses such as the FeMnTiO and the EA Ising model.

The unified description of the ZFC magnetizations means that eqs.(3) and (2) are quantitatively consistent with the measured results on the $\mathrm{FeMnTiO}$ spin glass when $\tau_{0} \sim 10^{-12} \mathrm{~s}$ and the same value of $b_{\mathrm{f}}$ as that of the EA-SG model are used. It is, to our knowledge, the first case in the study on aging in spin glasses that such a quantitative agreement is obtained in the direct comparison of the experiment with the numerical simulation.

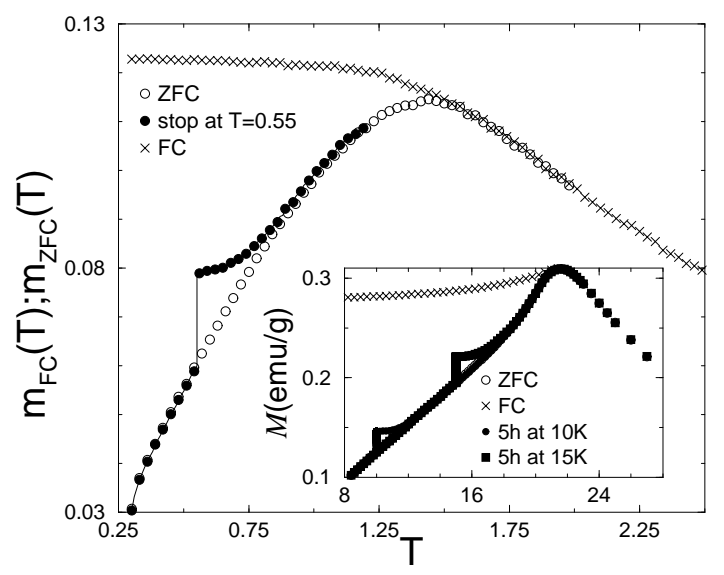

FIG. 1. The ZFC (o) and FC $(\times)$ magnetizations of the EA Ising SG model. The solid circles represent $m_{\mathrm{ZFC}}(T)$ with an intermittent stop of $10^{4} \mathrm{MCS}$ at $T=0.55$. In the inset we show the corresponding results measured in the Ising SG $\mathrm{Fe}_{0.50} \mathrm{Mn}_{0.50} \mathrm{TiO}_{3}$. The solid circles and squares represent $m_{\mathrm{ZFC}}(T)$ with an intermittent stop of 5 hours at $10.0 \mathrm{~K}$ and $15.0 \mathrm{~K}$, respectively.

We have measured the induced magnetization of the single crystal FeMnTiO sample with $T_{\mathrm{c}}=21.5 \mathrm{~K}$ 10 by applying the field parallel to the hexagonal $c$-axis of the sample (see [2] for further experimental details). The numerical simulations have been done on the 3D EA model with nearest neighbour interactions of mean zero and variance $J$ which is the unit of energy. We have used the heat-bath dynamics and have simulated several hundred samples with a linear size $L=24$ to make the error bars less than the size of the symbol in each plot. It is noted that the units of time and temperature used in the present work are respectively second and Kelvin in the experiment, and MCS and $J$, which we put unity below, in the simulation. Our processes measuring magnetizations in the experiment [simulation] are as follows. The system was first cooled from $50 \mathrm{~K}$ to $5 \mathrm{~K}[2.5 \mathrm{~J}$ to $0.3 \mathrm{~J}]$ at a rate of $1.0 \mathrm{~K} / \mathrm{min}[0.05 \mathrm{~J} / 10 \mathrm{MCS}]$ under zero field. Once the lowest temperature was reached the field of 100 Oe $[0.2 J]$ was applied and the sample was heated stepwise with a step of height $0.2 \mathrm{~K}[0.01 \mathrm{~J}]$ and length $100 \mathrm{~s}$
[100MCS]. The last 13s [10MCS] of the latter were used to measure $m_{\mathrm{ZFC}, T[\tau]}\left(t_{a}, t_{\mathrm{w}}\right)$ which we regard as the ZFC magnetization at that temperature, $m_{\mathrm{ZFC}}(T)$. At $50 \mathrm{~K}$, in the experiment, we turned to decrease the temperature and measured the FC magnetization, $m_{\mathrm{FC}}(T)$, on cooling with the same rate and in the same field. In the simulation $m_{\mathrm{FC}}(T)$ was measured on cooling the sample under $h=0.2 J$ from $2.5 J$ down to $0.3 J$ with the same rate as for $m_{\mathrm{ZFC}}(T)$.

The ZFC and FC magnetizations as well as the one with an intermittent stop of the real and model Ising spin glasses, shown in Fig. 1, look qualitatively quite similar to each other. Particularly, $m_{\mathrm{FC}}(T)$ of both systems become nearly flat at $T$ sufficiently lower than $T_{\mathrm{c}}$. We can also observe the following common feature in $m_{\mathrm{ZFC}}(T)$ with an intermittent stop. During the stop $m_{\mathrm{ZFC}}(T)$ increases, and when the heating is restarted, it continues to increase. However it increases significantly much slowly than $m_{\mathrm{ZFC}}(T)$ without the stop, and is caught up by the latter at a certain $T$ below $T_{\mathrm{c}}$.

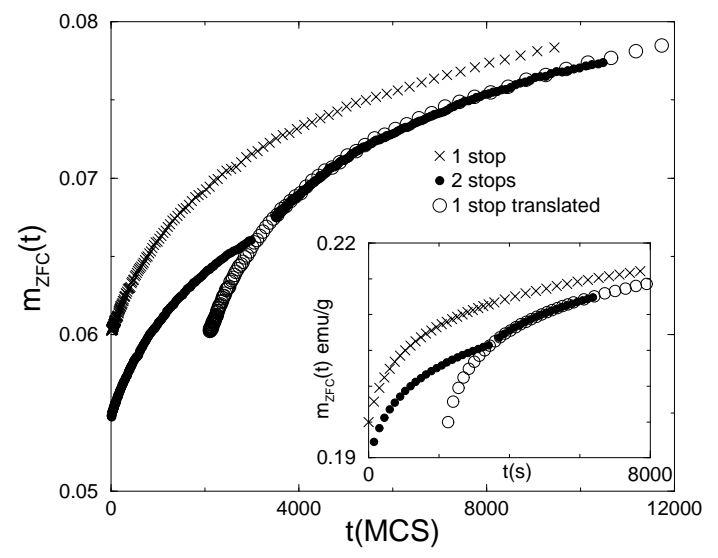

FIG. 2. Comparison of $m_{\mathrm{ZFC}, T[\tau]}\left(t_{a}, t_{\mathrm{w}}\right)$ with one and two intermittent stops in the EA model (main frame) and the FeMnTiO (inset) [2]. Here they are denoted as $m_{\mathrm{ZFC}}(t)$ (see the text).

In the inset [main frame] of Fig. 20 demonstrate the most interesting observation in our new protocol. It is the comparison of the explicit time evolutions of $m_{\mathrm{ZFC}, T[\tau]}\left(t_{a}, t_{\mathrm{w}}\right)$ of the FeMnTiO spin glass [the EA SG model] with one intermittent stop at $T=15.0 \mathrm{~K}[0.55 \mathrm{~J}]$ and two stops at $T=14.6 \mathrm{~K}$ by 1 hour and at $T=15.0 \mathrm{~K}$ $[T=0.50 J$ by $3000 \mathrm{MCS}$ and at $T=0.55 J]$. Here we rewrite them as $m_{\mathrm{ZFC}}(t)$ since we are interested in their time evolution. The origin of the time $t$ is set at the instance when the system is heated up to $15.0 \mathrm{~K}[0.55 \mathrm{~J}]$ for the 1-stop process and to $14.6 \mathrm{~K}[0.50 \mathrm{~J}]$ for the 2-stop process. It is seen that, when the 1-stop curve (crosses) is shifted to the right by $2250 \mathrm{~s}$ [2100 MCS] (open circles), it almost perfectly collapses to the branch of $T=15.0 \mathrm{~K}$ $[0.55 \mathrm{~J}]$ of the 2 -stop curve (solid circles). In other words, once $m_{\mathrm{ZFC}, T[\tau]}\left(t_{a}, t_{\mathrm{w}}\right)$ of two different thermal processes coincide with each other, their subsequent evolutions under a common condition of $T$ and $h$ are also identical. 
Keeping in mind that these processes have a common $R\left(t_{\mathrm{w}}\right)$, or a common thermal history before $h$ is applied, the results on both the real and model spin glasses are consistent with eq.(3) derived from the droplet picture.

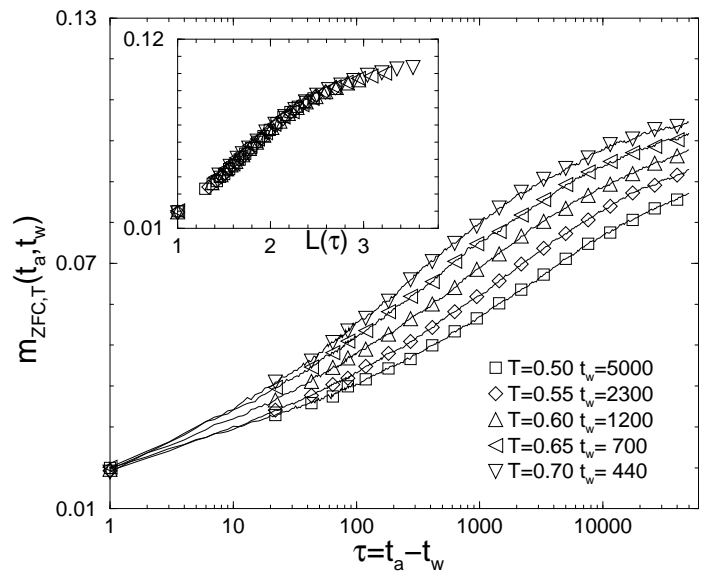

FIG. 3. Evolution of the isothermal ZFC magnetization of the EA model at different temperatures but starting from a common $R_{T}\left(t_{\mathrm{w}}\right)(\simeq 2.1)$. In the inset, the same data are plotted against $L_{T}(\tau)$ evaluated by eq.(2).

To ascertain the scaling expression of eq.(3), and to demonstrate that the $T$-dependence of the scaling function $\chi_{\mathrm{ZFC}}\left(L(\tau), R\left(t_{\mathrm{w}}\right)\right)$ comes out only through that of $L(\tau)$, we have performed the simulation of isothermal ZFC magnetization processes at various temperatures but by choosing the waiting time $t_{\mathrm{w}}$ after the instantaneous quench such that $R_{T}\left(t_{\mathrm{w}}\right)$ given by eq.(11) become the same for all temperatures. The results are shown in Fig. 3 where $m_{\mathrm{ZFC}, T}\left(t_{a}, t_{\mathrm{w}}\right)$ are plotted against the time $\tau$ after the field $h=0.2$ is switched on. The chosen $R_{T}\left(t_{\mathrm{w}}\right)$ is $\simeq 2.1$ and the corresponding $t_{\mathrm{w}}$ at each temperature are indicated in the figure.

The evolutions of $m_{\mathrm{ZFC}, T}\left(t_{a}, t_{\mathrm{w}}\right)$ in Fig. 3 depend on $T$ except at $\tau \simeq 1$. When, however, the same data are plotted against $L_{T}(\tau)$ evaluated by eq.(2), they are nicely superposed upon each other as shown in the inset of Fig. 3. This result strongly supports the scaling expression of eq.(3i) in the following two respects: the thermal history before the field is applied is in fact renormalized to $R\left(t_{\mathrm{w}}\right)$, and the size $L_{T}(\tau)$ of droplet excitations which are dominant in the time scale $\tau$ after the field is applied is properly given by eq.(2). We also note that, within the accuracy of the present numerical analysis, the scaling behavior is ascertained not only in the quasiequilibrium regime, where $L_{T}(\tau) \lesssim R_{T}\left(t_{\mathrm{w}}\right)$ or $\tau \ll t_{\mathrm{w}}$, as predicted by the droplet theory [3,9], but also in the outof-equilibrium, or aging regime, where $L_{T}(\tau) \gtrsim R_{T}\left(t_{\mathrm{w}}\right)$ or $\tau \gg t_{\mathrm{w}}$. In the latter regime $L_{T}(\tau)$ plays a role of the mean domain size at time $t_{a}$ in place of $R_{T}\left(t_{\mathrm{w}}\right)$.

Lastly let us extend the scaling analysis done above to our new experimental protocol in which the temperature is changed. For this purpose it is important to note that, so long as the system is in the SG phase, the mean do- main size $R\left(t_{\mathrm{w}}\right)$ of the EA model analyzed numerically ever grows continuously even when the temperature is changed discontinuously [11]. It is then natural for us to expect that this is also the case for $L(\tau)$ under a fixed field $h$, so that we may successively use eq.(2) as follows. Suppose that temperature is changed as $T_{i}=T_{i-1}+\Delta T_{i}$ and a period of time $d \tau_{i}$ is spent at each temperature $T_{i}$. We assume that the length scale achieved after the step at $T_{i}$ is given as

$$
L_{i}=\left(\tau_{i}^{\mathrm{eff}}+d \tau_{i}\right)^{b_{\mathrm{f}} T_{i} / T_{\mathrm{c}}},
$$

where $\tau_{i}^{\text {eff }}$ is an effective elapsed time to take into account the thermal history up to the instance just before the temperature is changed to $T_{i}$. It is the time needed to achieve the length scale $L_{i-1}$ reached after the $(i-1)$-th step by isothermal aging at $T_{i}$,

$$
\left(\tau_{i}^{\text {eff }}\right)^{b_{\mathrm{f}} T_{i} / T_{\mathrm{c}}}=L_{i-1} .
$$

Combining these we obtain a recursion formula for $L_{i}$ and/or $\tau_{i}^{\text {eff }}$ which is solved for any given temperature process with $L(\tau=0)=0$.

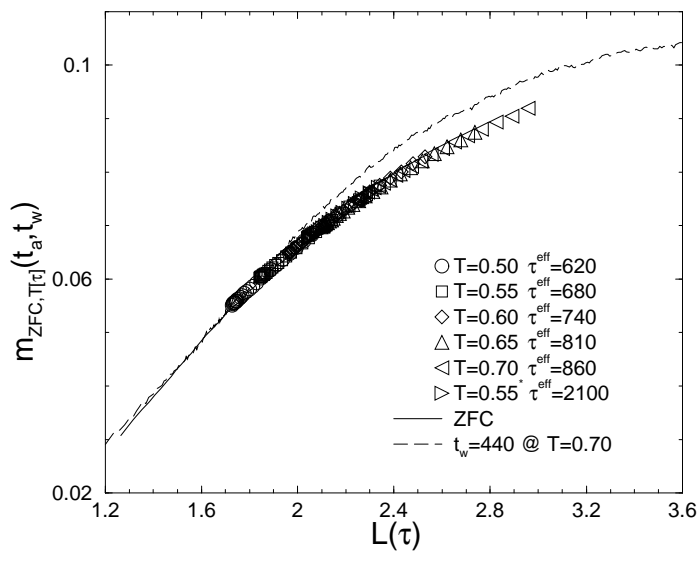

FIG. 4. Scaling plot of $m_{\mathrm{ZFC} T[\tau]}\left(t_{a}, t_{\mathrm{w}}\right)$ of the EA model against $L(\tau)$ evaluated by eqs. (4) and (5). The solid line represents the one without intermittent stop, while the symbols are those at the stop at various temperatures $T$ where $\tau^{\text {eff }}$ is the effective elapsed time at the instance when the system just reaches $T$. The data at $T=0.55$ with $\tau^{\text {eff }}=2100 \mathrm{MCS}$ represent the second stop in the 2 -stop process. The dashed line is one of the curves shown in the inset of Fig. 3.

We have first applied the above-mentioned analysis to $m_{\mathrm{ZFC}, T[\tau]}\left(t_{a}, t_{\mathrm{w}}\right)$ of the EA model in the processes with $T$-changes shown in Figs. 1 and 2. The results are shown in Fig. 1. All $m_{\mathrm{ZFC}, T[\tau]}\left(t_{a}, t_{\mathrm{w}}\right)$ nicely lie on a universal curve, indicating that the scaling of eq.(3)), combined with the above analysis on $L(\tau)$, does work well. In the figure we also draw one of the curves in the isothermal process shown in the inset of Fig. 3. It has the different thermal history before the field is applied, i.e., the different $R\left(t_{\mathrm{w}}\right)$, from those of the $T$-change process. It significantly deviates from the universal curve for the latter as expected from eq.(3). 
Surprisingly, the $m_{\mathrm{ZFC}, T[\tau]}\left(t_{a}, t_{\mathrm{w}}\right)$ curves measured in the FeMnTiO spin glass and shown in the inset of Figs. 1 and 2 obey the same scaling law as those of the EA model.

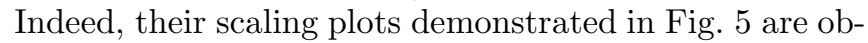
tained by making use of eq.(2) with the identical exponent of $b_{\mathrm{f}} T / T_{\mathrm{c}}$ but with $\tau_{0}=10^{-9} \mathrm{~s}$ and $10^{-12} \mathrm{~s}$, instead of $\tau_{0}=1$ MCS for the EA model. The two portions with the dense data points at around $m_{\mathrm{ZFC}, T[\tau]}\left(t_{a}, t_{\mathrm{w}}\right) \simeq 0.14$ and 0.20 represent the results with intermittent stops at $9.6 \mathrm{~K}, 10.0 \mathrm{~K}$ and at $14.6 \mathrm{~K}, 15.0 \mathrm{~K}$, respectively. At a glance we see that the scaling with $\tau_{0}=10^{-12} \mathrm{~s}$ is the better. We have not performed further analysis on the sensitivity of the collapse of the curves to the parameter values, because the evolution law of eq.(2) or (11) already involves certain uncertainty. For example, $R_{T}\left(t_{\mathrm{w}}\right)$ extracted by the ac susceptibility in a sufficiently wide frequency range is better expressed by a function of $\ln t_{\mathrm{w}}$ [12]. Furthermore we are faced to similar but more serious problems in the temperature range close to $T_{\mathrm{c}}$, such as the crossover to the critical regime [3,13], whose analysis has been omitted in the present work. However we consider that the scaling results obtained above are enough for us to claim that the droplet picture expressed by eq.(3) is consistent with the aging behavior of the ZFC magnetization in the FeMnTiO Ising spin glass, and that the length scale involved in the process is about a hundred lattice distances or less. They also imply the continuous growth of $L(\tau)$ and so $R\left(t_{\mathrm{w}}\right)$, without suffering from the chaotic effect [14], at least in the present experimental protocol.

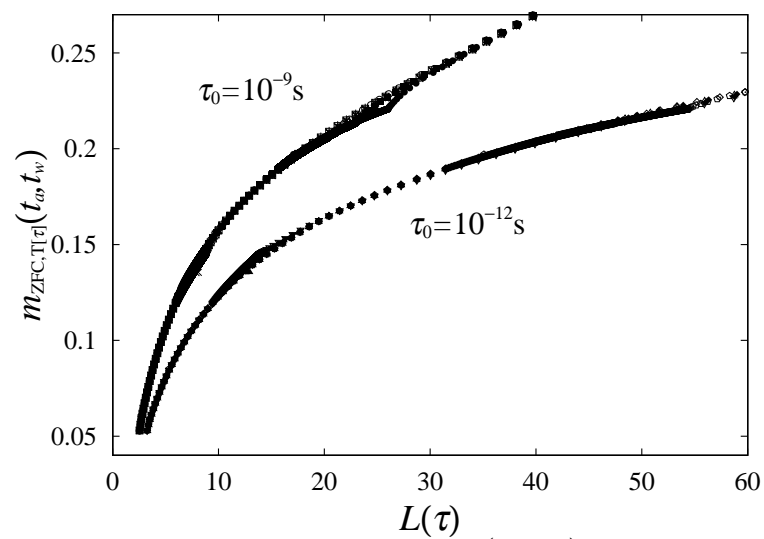

FIG. 5. Scaling plots of $m_{\mathrm{ZFC}, T[\tau]}\left(t_{a}, t_{\mathrm{w}}\right)$ of the FeMnTiO spin glass shown in the inset of Figs. 1 and 2. The abscissa $L(\tau)$ is evaluated by eq. (2) with $\tau_{0}=10^{-9} \mathrm{~s}$ and $10^{-12} \mathrm{~s}$.

Another interesting result of the present work is that the evolution law of $L_{T}(\tau)$ given by eq.(2), or the growth law of $R_{T}\left(t_{\mathrm{w}}\right)$ given by eq.(位), almost coincides with the growth law of the SG coherence length, $\xi_{T}(t)$, which Joh et al. 15, 16. has extracted from the thermoremanent magnetization (TRM) of the spin glasses CuMn, AgMn and $\mathrm{CdIn}_{0.3} \mathrm{Cr}_{1.7} \mathrm{~S}_{4}$, though none of them is an Ising spin glass. Their results imply that the evolution law of $L_{T}(\tau)$ depends on the field at least in the the crossover region between the quasi-equilibrium and the aging regimes. In the present work, however, such a field effect has been neglected regarding it as a higher order effect.

To conclude we have demonstrated that the ZFC magnetizations measured in both real and model Ising spin glasses are described by a common scaling expression which is consistent with the droplet picture on the SG aging dynamics. The result indicates that the numerical simulation on the model spin glass supplies us not only qualitative but also quantitative information on aging phenomena in real spin glasses.

This work is supported by Grant-in-Aids for International Scientific Research Program (\#10044064) and for Scientific Research Program (\#10640362), from the Ministry of Education, Science, Sports and Culture, Japan. The present simulation has been performed by the computing facilities at Supercomputer Center, Institute for Solid State Physics, University of Tokyo.

[1] L. Lundgren, P. Svedlindh, P. Nordblad and O. Beckman, Phys. Rev. Lett. 51, 911 (1983).

[2] A. Ito, A. Tobo, N. Onchi and J. Satooka, to appear in J. Phys. Soc. Jpn. Suppl.

[3] D.S. Fisher and D.A. Huse, Phys. Rev. B 38, 373 (1988).

[4] D.A. Huse, Phys. Rev. B 43, 8673 (1991).

[5] J. Kisker, L. Santen, M. Schreckenberg and H. Rieger, Phys. Rev. B 53, 6418 (1996).

[6] E. Marinari, G. Parisi, F. Ricci-Tersenghi and J.J. RuizLorenzo, J. Phys. A 31, 2611 (1998).

[7] T. Komori, H. Yoshino and H. Takayama, J. Phys. Soc. Jpn. 68, 3387 (1999).

[8] P.O. Mari and I.A. Campbell, Phys. Rev. E 59, 2653 (1999).

[9] T. Komori, H. Yoshino and H. Takayama, J. Phys. Soc. Jpn, 69, 1192 (2000).

[10] A. Ito, H. Aruga, E. Torikai, M. Kikuchi, Y. Syono and H. Takei, Phys. Rev. Lett. 57, 483 (1986).

[11] T. Komori, H. Yoshino and H. Takayama, to appear in J. Phys. Soc. Jpn. Suppl.

[12] J. Hammann, M. Ocio and E. Vincent, in "Relaxation in Complex Systems and Related Topics", edited by I.A. Campbell and O. Giovanella (Plenum Press, New York, 1990).

[13] K. Hukushima, H. Yoshino and H. Takayama, to appear in Prog. Theor. Phys. Suppl.

[14] A. J. Bray and M. A. Moore, Phys. Rev. Lett. 58, 57 (1987).

[15] Y. G. Joh, R. Orbach, G. G. Wood, J. Hammann and E. Vincent, Phys. Rev. Lett. 82, 438 (1999).

[16] Y. G. Joh, R. Orbach, G. G. Wood, J. Hammann and E. Vincent, to appear in J. Phys. Soc. Jpn. Suppl. 